\title{
Widersprüchliche Dynamiken im deutschen Arbeitsmarkt
}

Vom „kranken Mann Europas“ zum Beschäftigungswunder? Zunehmende Prekarisierung oder Stabilisierung von Beschäftigungsverhältnissen selbst in Krisenzeiten? Polarisierte Parallelwelten in einem grundlegend „dualisierten“ Erwerbssystem oder zwei Seiten derselben Medaille? Der Beitrag versucht, die widersprüchlichen Erscheinungen am deutschen Arbeitsmarkt in einen Zusammenhang zu bringen und so Forschungen anzuregen, die die Grenzen von Disziplinen und eingefahrenen Betrachtungsweisen überschreiten.

\section{Einleitung}

Der folgende Beitrag stellt den Versuch dar, Entwicklungen in einem Zusammenhang zu diskutieren, die üblicherweise in unterschiedlichen Fachdisziplinen, unverbundenen Diskursarenen und getrennten Politikfeldern verhandelt werden. Die Ausführungen haben unvermeidlich hypothetischen Charakter, weil sie Forschungsaufgaben umreißen, die noch nicht geleistet sind und die bei inkrementeller, pfadabhängiger Entwicklung der Forschung voraussichtlich auch nicht geleistet werden. Das liegt nicht zuletzt auch daran, dass bestehende Forschungsperspektiven jeweils ihre eigenen, institutionell wie methodisch in spezifischer Weise beschränkten Datenreihen und Paneldatensätze benutzen müssen. Diese wirken wie fest installierte Teleskope: Man kann mit methodischen Mitteln die Brennweite und damit die Blicktiefe verändern, aber nicht die Blickrichtung, die jeweils alternativ auf Betriebe, Erwerbstätige, Haushalte, Leistungsempfänger einer bestimmten Kategorie usw. ausgerichtet ist. Nur durch Überschreitung dieser Grenzen und damit durch eine mit Daten lediglich punktuell und mosaikhaft illustrierbare Argumentation lassen sich größere Zusammenhänge herstellen, deren Überprüfung freilich aussteht.

\section{Der Arbeitsmarkt wird flüssiger: mehr Übergänge aus Arbeitslosigkeit in Erwerbstätigkeit}

Die Situation des deutschen Arbeitsmarktes stellt sich derzeit sehr widersprüchlich dar. Auf den ersten Blick handelt es sich um eine für viele unerwartete und im öffentlichen Bewusstsein noch nicht vollständig angekommene Erfolgsgeschichte. Die Arbeitslosenquote nach der amtlichen Zählung ist auf das Niveau von 1992 gesunken. Die Zahl der Erwerbstätigen befindet sich ebenso auf Rekordniveau wie die Zahl der Arbeitnehmerinnen und Arbeitnehmer; die sozialversicherungspflichtige Beschäftigung hat in etwa wieder das Niveau von Anfang der 1990er Jahre erreicht, ${ }^{1}$ wobei die notwendige Differenzierung dieser Aussagen nach Beschäftigungsform bereits auf grundlegende strukturelle Veränderungen verweist (Keller/Seifert 2011).

Entgegen der weit verbreiteten Betrachtung von Arbeitslosigkeit und Erwerbstätigkeit als kommunizierenden Röhren bedeutet die Abnahme der Arbeitslosigkeit kein außergewöhnliches Erwerbswachstum. Die Erwerbstätigkeit hat zwar im Aufschwung 2005 bis 2008 zugenommen (und durch die Finanz- und Wirtschaftskrise kaum gelitten), doch bezogen auf seine Dauer war dieser Aufschwung wohl nicht erwerbswirksamer als frühere (Horn et al. 2008), und das gesamtwirtschaftliche Arbeitsvolumen von 2008 lag nach den Berechnungen des Instituts für Arbeitsmarkt- und Berufsforschung (IAB) noch leicht unter dem von 2000. Das entscheidend Neue liegt vielmehr darin, dass das Verhältnis von Zunahme der Erwerbstätig- keit und Abbau der Arbeitslosigkeit günstiger geworden ist. ${ }^{2}$ Die Abgänge aus Arbeitslosigkeit in Erwerbstätigkeit, ${ }^{3}$ die von Mitte der 1990er Jahre bis 2004 kaum noch auf Veränderungen der Nachfrage reagiert hatten, haben sich wieder an den Nachfragezyklus angekoppelt (Abbildung 1). ${ }^{4}$

Ebenfalls hat zum aktuellen Rückgang der Arbeitslosigkeit beigetragen, dass der umgekehrte Strom, die Zugänge aus Erwerbstätigkeit in Arbeitslosigkeit, nach Rekordwerten 2003/2004 (möglicherweise Vorzieheffekte in Antizipation der Hartz-Reformen, vgl. Dlugosz et al. 2009) ab 2005 erheblich zurückgegangen und in der Wirtschafts- und Finanzkrise ab 2008 nur kurzzeitig und moderat angestiegen sind (Abbildung 1). Die erweiterte Kurzarbeitsregelung hat zu dieser Stabilisierung beigetragen, ist aber nur zum geringeren Teil direkt ursächlich dafür (Möller 2010;

\footnotetext{
1 Zeitreihe der Bundesagentur für Arbeit (BA) unter http://statistik.arbeitsagentur.de/Statistikdaten/ Detail/Aktuell/iiia6/sozbe-zr-svb-74/zr-svb-74d-0-xls.xls, abgerufen am 27.09.2011.

2 Dieses gilt auch dann, wenn man für die Sondereffekte von "Hartz IV “ - zunächst rechnerische Steigerung der Arbeitslosigkeit, dann massenhafter Einsatz von "Arbeitsgelegenheiten“ - korrigiert (Knuth 2010).

3 „Erwerbstätigkeit “ enthält hier auch Arbeitsgelegenheiten und andere „Beschäftigung schaffende Maßnahmen“ (eine Trennung ist mit veröffentlichten BA-Daten leider nicht möglich), aber dieses gilt natürlich in beiden Richtungen des Übergangs.

4 Eine ähnliche Entwicklung konnte im Zehn-JahresAbstand 1996/2006 für mehrere andere westeuropäische Länder gezeigt werden (Koslowski et al. 2011).
}

Matthias Knuth, Prof. Dr., ist Soziologe und forscht am Institut Arbeit und Qualifikation (IAQ) der Universität Duisburg-Essen. Arbeitsschwerpunkte: Arbeitsmarkt, Arbeitsmarktpolitik, Altersübergang. e-mail: matthias.knuth@uni-due.de 


\section{Abb. 1: Übergänge zwischen Arbeitslosigkeit und Erwerbstätigkeit und Bestand an Erwerbstätigen 1998 bis 2010 - in 1.000 -}

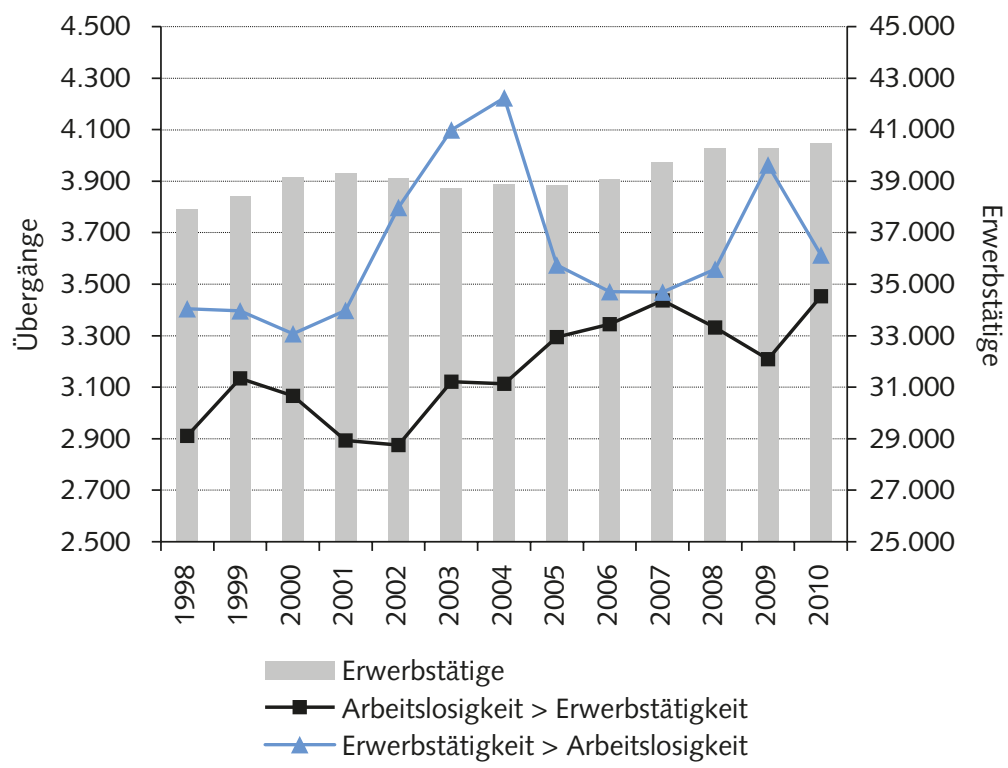

Quelle: Bundesagentur für Arbeit 2011a und frühere Jahrgänge dieser Reihe; Darstellung des Autors.

WSI MITTEILUNGEN

Herzog-Stein/Seifert 2010). Indirekt hat die Kurzarbeit zu einem geschäftspolitischen Klima beigetragen, in dem die Ankündigung von Massenentlassungen - anders als in vorausgegangenen Zyklen - nicht mehr als Ausweis vorausschauender Unternehmenspolitik galt. Vermutlich wirken die Erwartung von Fachkräftemangel und die weitgehende Beseitigung der Attraktivität von vorruhestandsähnlichen Regelungen, soweit sie mit dem Bezug von Lohnersatzleistungen verbunden waren, dahingehend zusammen, dass die Betriebe im Durchschnitt mit Entlassungen in die Arbeitslosigkeit zurückhaltender geworden sind. ${ }^{5}$ Dieses gilt jedenfalls im Vergleich von 2009 zu 2004.

Mit Blick auf Arbeitslosigkeit lässt sich schlussfolgern, dass der Arbeitsmarkt flüssiger geworden ist. Dabei sind die Chancen, Arbeitslosigkeit zugunsten einer Erwerbstätigkeit zu verlassen, stärker gewachsen als das Risiko, durch Verlust einer Erwerbstätigkeit arbeitslos zu werden, gleichwohl haben beide im Trend zugenommen. ${ }^{6}$ Zweifellos müssen wir also von „Drehtüreffekten“ ausgehen (Koller/Rudolph 2011), aber es gehen doch mehr in die Drehtür hinein als wieder herauskommen.

samtfluktuation am Arbeitsmarkt $(A b$ bildung 2), obwohl darin natürlich die im vorigen Abschnitt betrachteten Übergänge in und aus Arbeitslosigkeit weitgehend enthalten sind. ${ }^{7}$ Das Verhältnis von Nachfrage und Angebot am Arbeitsmarkt ist günstiger geworden; auch „die Stille Reserve“ ist stark gesunken. Diese für die Anbieter von Arbeitskraft günstiger gewordenen Knappheitsverhältnisse schlagen sich jedoch weitaus weniger als in früheren Wachstumsperioden in einer Zunahme von Betriebswechseln der Beschäftigten nieder.

Wie bereits festgestellt, haben Arbeitslose einen höheren Anteil als in den letzten Aufschwüngen daran gehabt, die betriebliche Arbeitskräftenachfrage zu befriedigen. Die grundlegende Veränderung des Arbeitsmarktregimes durch die Hartz- schäftigte hingegen scheinen zunehmend die Risiken einer „Reise über den Arbeitsmarkt" zu scheuen, die ja dazu führt, dass an die Dauer der Betriebszugehörigkeit gebundene gesetzliche und tarifliche Kündigungsschutzpositionen und andere Rechte neu erworben werden müssen. Stattdessen sind sie für die Aufrechterhaltung ihres Beschäftigungsverhältnisses verstärkt zu Zugeständnissen bereit (ebd.).

Wenn eine offene Stelle durch Einstellung einer aktuell nicht beschäftigten Person statt durch Abwerbung einer beschäftigten Person besetzt wird, entsteht keine Vakanzkette; die Stellenbesetzung in dem einen Betrieb löst keine Vakanz in einem anderen Betrieb und damit keine erneute Stellenbesetzung (und womöglich weitere Abwerbung) aus. Solche Vakanzketten wirken im Aufschwung als Multiplikator der Fluktuation, die deshalb weitaus stärker zunimmt als die Nettogröße des zusätzlichen Arbeitskräftebedarfs (Schettkat 1996). Der erfreuliche Abbau der Arbeitslosigkeit durch eine erhöhte Abgangsrate in Erwerbstätigkeit hat folglich eine Kehrseite, die im Zeitalter der „aktivierenden Arbeitsmarktpolitik“ wenig diskutiert wird: Nicht nur, dass die Qualität der Arbeitsplatzangebote sinkt - ganz im Sinne des Theorems von der „industriellen Reservearmee“ oder der umgekehrten Bewertung dieses Phänomens in der "Insider-Outsider-Theorie“ (Lindbeck/Snower 1988) -, sondern auch, dass eine Aufschwungphase weniger Reallokation auf dem Arbeitsmarkt ausReformen (Knuth 2006) erklärt, warum Arbeitslose eher als früher bereit sind, Arbeit auch zu ungünstigeren Konditionen anzunehmen (Rebien/Kettner 2011). Be-
5 Zwar kommt hinzu, dass Anpassungsleistungen der betrieblichen Beschäftigung zunehmend auf Zeitarbeitskräfte, befristet Beschäftigte und Subunternehmer abgewälzt werden; soweit dieses aber zu Eintritten in Arbeitslosigkeit führt, sind diese Effekte natürlich in Abbildung 1 enthalten.

6 Dass die Übergänge aus Erwerbstätigkeit in $\mathrm{Ar}$ beitslosigkeit stets zahlreicher sind als die umgekehrten Übergänge, während die Arbeitslosigkeit trotzdem sinkt, sollte hier nicht irritieren, da es mit Ruhestand und Rückzug vom Arbeitsmarkt wesentliche, aus dieser Betrachtung ausgeblendete Übergänge gibt, durch die Arbeitslosigkeit verlassen wird.

7 Derartige Fluktuationsdaten sind leider nur für die sozialversicherungspflichtige Beschäftigung verfügbar. Das denkbare Argument, dass die stärkere Bewegung bei Arbeitslosen allein auf nicht sozialversicherungspflichtige Beschäftigung zurückzuführen sei, ist jedoch nicht stichhaltig, da die in den Abbildungen 1 und 2 dargestellten Übergänge eine Erwerbstätigkeit von mindestens 15 Stunden voraussetzen, womit ein großer Teil der Mini-Jobs ebenso herausfällt wie selbstständige Tätigkeit in kleinem Umfang. 
löst. Anders gesagt, nach 25 Jahren der Bemühungen zur „Flexibilisierung“ des Arbeitsmarktes ${ }^{8}$ stellt sich dieser als insgesamt weniger flüssig dar. Obwohl oder auch gerade weil der Anteil von Arbeitsverhältnissen gewachsen ist, die von vornherein mit einem Verfallsdatum versehen sind, kommt es insgesamt nicht zu mehr, sondern zu weniger Übergängen am Arbeitsmarkt: Im Gegensatz zur Steigerung der Übergänge zwischen Arbeitslosigkeit und Erwerbstätigkeit ist der Arbeitsmarkt insgesamt trotz gewachsener Nachfrage zähflüssiger geworden.

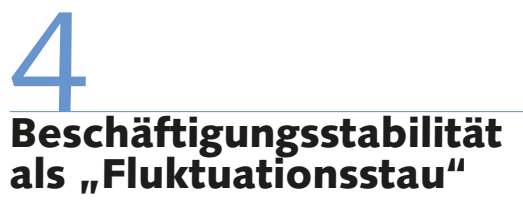

Die zunehmende Zähflüssigkeit des Arbeitsmarktes insgesamt schlägt sich auch darin nieder, dass - ganz im Gegensatz zur verbreiteten Wahrnehmung zunehmender Volatilität oder Prekarität - die durchschnittliche unabgeschlossene Betriebszugehörigkeit der Beschäftigten zunimmt (Erlinghagen 2010; Rhein 2010). Nun sind dauerhafte Bindungen von Betrieben und Arbeitnehmern grundsätzlich gewiss kein Nachteil, sondern ein wesentlicher Bestandteil des eher auf interne als auf externe Flexibilität setzenden deutschen Produktionsmodells, das gerade in und nach der Krise 2008 - 2010 seine Leistungsfähigkeit wieder unter Beweis gestellt hat. Wenn jedoch gleichzeitig die Angst vor Arbeitsplatzverlust steigt (Erlinghagen/ Wagner 2010) und die Arbeitszufriedenheit allgemein (Matiaske/Mellewigt 2001) mit zunehmender Betriebszugehörigkeit (Hauser et al. 2008) und mit zunehmendem Alter sinkt (Bohulsky et al. 2011; Fietze 2011), so dürfte es sich zu wachsenden Teilen um Betriebsbindungen aus Mangel an Alternativen handeln. Die subjektiv wahrgenommene Möglichkeit freiwilliger Mobilität und die Aussicht, dadurch berufliche Verbesserungen zu erreichen, sind ein wesentlicher Ausdruck persönlicher Freiheit, und sie tragen zum Selbstbewusstsein und zur Zufriedenheit auch derjenigen bei, die aktuell keinen Gebrauch davon machen.

Doch Beschäftigungswechsel führen heute seltener zu Verbesserungen als früher (Trischler/Kistler 2010). Wenn Senioritätsentlohnung in Deutschland offenbar

\section{Abb. 2: Fluktuationsration sozialversicherungspflichtiger Beschäftigung (Eintrittsraten, Austrittsraten, Gesamtfluktuation) 1976 bis 2009 - in \% -}

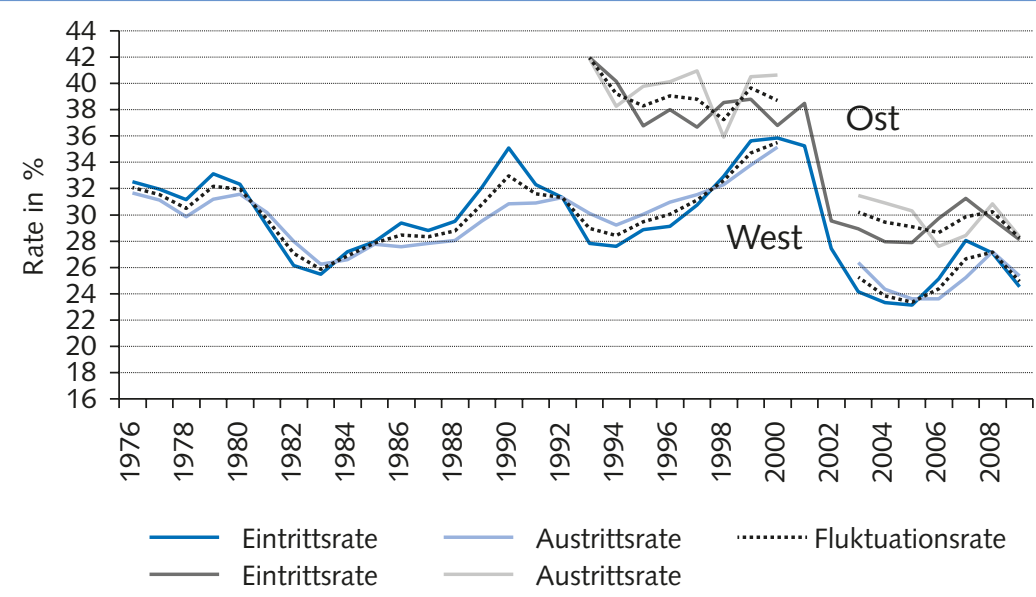

Quelle: Erlinghagen 2010; Fortschreibung nach BA-Statistik; Darstellung des Autors.

WSI MITEELUNGEN

eine viel geringere Rolle spielt als in importierten Modellwelten gemeinhin angenommen (Fachinger/Himmelreicher 2007; Orlowski/Riphahn 2008; Orlowski/Riphahn 2011), dann ist es weniger der noch zu erwartende „Aufzug nach oben“ im gegenwärtigen Betrieb, sondern der „Aufzug nach unten" im Falle eines Betriebswechsels, der Arbeitnehmer an Beschäftigungsverhältnissen festhalten lässt, die ihren Erwartungen und Potenzialen nicht entsprechen und ihrem Wohlbefinden nicht dienlich sind. Trotz allgegenwärtiger Klagen über Fachkräftemangel waren offenbar die betrieblichen Einstiegskonditionen im Durchschnitt nicht so, dass ein Wechsel verlockend erschien. Steigende Anteile von Befristungen unter den Neueinstellungen (Hohendanner 2010), Deckung betrieblichen Zusatzbedarfs zunehmend über die Zeitarbeit, zunehmende Lohnspreizung (Eichhorst et al. 2009), wachsende Anteile von Niedriglohnbeschäftigung (Kalina/ Weinkopf 2010) und die Ausgliederung betrieblicher Wachstumsbereiche in rechtlich eigenständige Einheiten mit schlechteren Konditionen geben Anhaltspunkte, warum Arbeitgeberwechsel für Beschäftigte weniger attraktiv sind als früher.

Der von Boerner/Schramm (1998) bereits für die 1990er Jahre vermutete „Fluktuationsstau“ dürfte sich im ersten Jahrzehnt des neuen Jahrtausends verschärft haben: Einerseits nahmen Arbeitslose vermehrt unterwertige Beschäftigung an (Achatz/Trappmann 2009), andererseits hatten unterwertig Beschäftigte auch bei steigender Arbeitsnachfrage weniger Mög- lichkeiten der Aufstiegsmobilität. Das gilt sowohl hinsichtlich des Einsatzes im bestehenden Beschäftigungsverhältnis wie auch im Hinblick auf die Segmentierung von Beschäftigungsformen: Atypische Formen der Beschäftigung erweisen sich eher als Sackgassen denn als Sprungbretter, und das gilt auch für Qualifizierte. Die wieder einmal aktuelle Diskussion über Fachkräftemangel ist auch vor dem Hintergrund dieser Fehlallokation von Arbeitskraft zu sehen.

\section{Das neue Arbeitsmarktre- gime: Marginalisierung der Arbeitslosenversicherung}

Die in der Öffentlichkeit übliche Beurteilung der Arbeitsmarktsituation allein aufgrund von Arbeitslosenzahlen ist unzureichend. Mit der „Grundsicherung für Arbeitsuchende“ (SGB II) wurde ein Leistungssystem geschaffen, dessen Zugehörigkeitsvoraussetzung nicht die Arbeitslosigkeit, sondern die Hilfebedürftigkeit trotz zumindest residualer Erwerbsfähigkeit ist. Die Zahl der „erwerbsfähigen Leistungsberechtigten" ist seit ihrem Höchststand im April 2006 bis zum April 2011 lediglich um $13 \%$ gesunken, während die Zahl der Arbeitslosen im gleichen Zeitraum um rund

\footnotetext{
8 Eine genaue Datierung ist natürlich unmöglich - hier wird das Beschäftigungsförderungsgesetz von 1985 als Referenzpunkt genommen.
} 
$36 \%$ abnahm. Im April 2011 wurden nur noch knapp $46 \%$ der „erwerbsfähigen Leistungsberechtigten “ (ArbeitslosengeldII-Beziehende) als Arbeitslose gezählt; im Jahre 2006 waren es noch 51,5\%.

Die in Abschnitt 2 festgestellte Verflüssigung der Arbeitslosigkeit verteilt sich sehr ungleich zwischen den „Rechtskreisen“ der Arbeitslosenversicherung (SGB III) und der Grundsicherung (SGB II). Zwar ergeben sich in beiden Rechtskreisen Steigerungen der auf die jeweils verbleibenden Arbeitslosen berechneten Abgangsraten in Erwerbstätigkeit, jedoch auf höchst unterschiedlichem Niveau und mit sehr unterschiedlicher Steigerungstendenz (Abbildung 3). Während die Abnahme auch der Langzeitarbeitslosigkeit öffentlich gefeiert wird, erweist sich der von administrativen Regeln der Arbeitslosenstatistik unberührte Leistungsbezug für einen relevanten Teil der Betroffenen als dauerhafter (Graf/Rudolph 2009). Die auf Befragungen beruhende und damit von administrativen Zuschreibungen weniger abhängige Langzeit-Erwerbslosenrate ${ }^{9}$ ist vom Rekordwert 2005 leicht unter den Wert von 2000 gesunken (auf 3,4\% im Jahr 2009), liegt damit aber immer noch über der Rate von 3,0 für die EU-15. Bei insgesamt günstiger Entwicklung der Arbeitslosigkeit gehört damit der Anteil der Langzeiterwerbslosen an allen Erwerbslosen in Deutschland mit $47 \%$ zu den höchsten in Europa (Bundesagentur für Arbeit 2011b), d.h. die Erwerbslosigkeitsdauer ist in Deutschland besonders stark polarisiert.

Bezogen auf erwerbsfähige Leistungsberechtigte statt auf Arbeitslose wird die
Integrationsquote in sozialversicherungspflichtige Beschäftigung für das Jahr 2010 mit lediglich $18,6 \%$ angegeben. ${ }^{10}$ Bei der Evaluationsforschung zum SGB II wurde zwischen zwei Befragungen im Abstand von knapp zwölf Monaten die Aufnahme einer Erwerbstätigkeit von $16 \%$ des Bestandes ermittelt (Brussig/Knuth 2011). In beiden Fällen ist zu beachten, dass dieses weder gleichzusetzen ist mit der Überwindung des Leistungsbezugs (die Erwerbstätigkeit deckt möglicherweise den Bedarf der Bedarfsgemeinschaft nicht) noch mit der Beendigung von Arbeitslosigkeit (bei Erwerbstätigkeit unter 15 Stunden pro Woche); macht man derartige Einschränkungen, ergeben sich noch niedrigere Werte. Der einzige bisher verfügbare Vergleich von Abgangsraten aus dem Bezug bedürftigkeitsgeprüfter Leistungen vor und nach der einschneidenden vierten Stufe der Hartz-Reformen findet, bezogen auf die Übergangswahrscheinlichkeit in Erwerbstätigkeit, keinen signifikanten Effekt (Fehr/Vobruba 2011). Die oben festgestellte Verflüssigung des Arbeitsmarktes für Arbeitslose trifft also in der Hauptsache für kurzzeitige Arbeitslosigkeit mit Versicherungsschutz zu; der Teil der Arbeitslosen, mit deren Lage die Hartz-Reformen gerechtfertigt wurden, bleibt überwiegend abgekoppelt.

Das verstärkte „Fordern und Fördern“ des SGB II wirkt also offenbar weniger auf diejenigen, die dieser Behandlung ausgesetzt sind, als auf jene, die sie vermeiden wollen. Das sind zum einen die noch versicherten Bezieherinnen und Bezieher von

\section{Abb. 3: Abgangsraten aus Arbeitslosigkeit in Erwerbstätigkeit* nach Rechtskreisen SGB III und SGB II 2005 bis 2010}

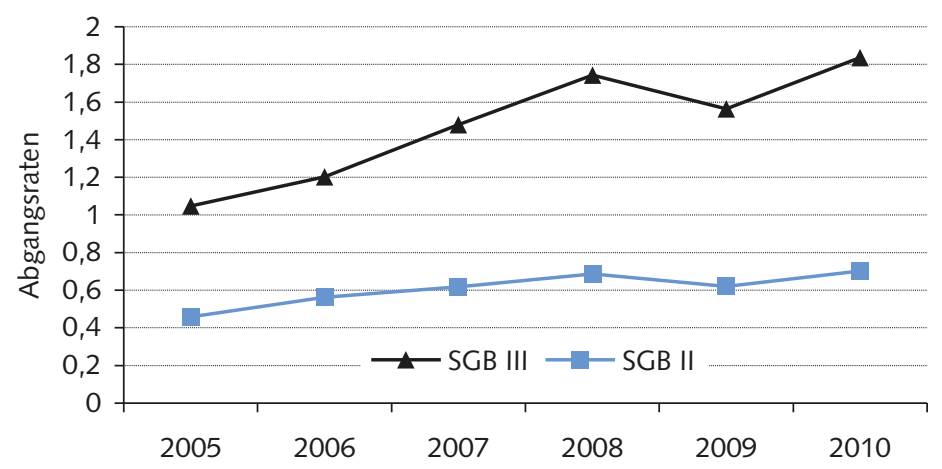

*Erwerbstätigkeit nach dieser BA-Statistik schließt geringfügige Beschäftigung, Arbeitsgelegenheiten und berufliche Selbstständigkeit ein; jedoch können Tätigkeiten unter 15 Stunden/Woche definitionsgemäß nicht zur Beendigung der Arbeitslosigkeit führen und sind daher nicht enthalten. Eine Beschränkung der Betrachtung auf "sozialversicherungspflichtige Beschäftigung im regulären Arbeitsmarkt " wäre wünschenswert, jedoch sind hierzu aus den Veröffentlichungen der BA keine längerfristigen Zeitreihen zu erschließen.

Quelle: Bundesagentur für Arbeit, Eingliederungsbilanzen; Berechnung und Darstellung des Autors ohne Daten der zugelassenen kommunalen Träger.

Arbeitslosengeld, die, wie bereits dargestellt, rascher und offenbar bereitwilliger eine Erwerbstätigkeit auch zu schlechteren Konditionen aufnehmen - mit dem positiven Effekt kürzerer abgeschlossener Arbeitslosigkeitsdauern und andererseits dem Risiko unterwertiger oder atypischer Beschäftigung. Zum anderen wirkt das neue Arbeitsmarktregime auf die Beschäftigten, die das Risiko von Arbeitslosigkeit trotz sinkender Arbeitslosenzahlen höher bewerten als vor den Hartz-Reformen, weil sie nur für kürzere Zeit mit Lohnersatzleistungen rechnen können, die am früheren Einkommen bemessen sind. Soweit sinkende Realverdienste im Falle des Arbeitsplatzverlustes zu nicht bedarfsdeckenden Ansprüchen auf Arbeitslosengeld und damit in den aufstockenden Bezug von Leistungen der Grundsicherung führen, gilt von Beginn der Arbeitslosigkeit das restriktivere, gegenüber der Qualität von Beschäftigung gleichgültige Aktivierungsregime des SGB II. Versicherungsbeiträge führen hier also nicht zu einer versicherungsförmigen Risikosicherung, weil das „untere“ Sicherungssystem das „obere“ dominiert. Für diejenigen, die sich in der „Drehtür“ von Arbeitslosigkeit und Beschäftigung befinden, bewirkt die Verkürzung der Rahmenfrist für den Erwerb von Ansprüchen auf Arbeitslosengeld einen tendenziellen Ausschluss von Versicherungsleistungen überhaupt. Von einer „Beschäftigungsversicherung" (Schmid 2008, 2011) sind wir also weiter entfernt als vor den Reformen - das neue Arbeitsmarktregime ist eher als eine Marginalisierung der Arbeitslosenversicherung zu kennzeichnen, die weitreichende Auswirkungen auch auf die Beschäftigten hat.

\section{Umfassenderer Zugriff auf Arbeitskraft}

In Anknüpfung an die lange Tradition dichotomer Konzeptualisierungen von betrieblichen Beschäftigungssystemen und Arbeitsmärkten (Kerr 1954; Doeringer/

\footnotetext{
9 Anteil der Langzeit-Erwerbslosen an den Erwerbspersonen (European Commission 2010).

10 Vgl. Informationsplattform SGB II, http://www. sgb2.info/kennzahlen/statistik, Abfrage für Januar 2011 am 27.09.2011. Diese Daten werden derzeit noch unter dem Vorbehalt eingeschränkter Aussagekraft veröffentlicht.
} 
Piore 1971; Lindbeck/Snower 1988), und vermutlich beeinflusst von der französischen Denktradition gesellschaftlicher Inklusion versus Exklusion, wird die Verschärfung der Grenzziehung zwischen Grundsicherung und Versicherung in „Bismarck'schen“ Wohlfahrtsstaaten von manchen Autoren zum Anlass genommen, eine grundlegende, nunmehr staatlich institutionalisierte „Dualisierung“ des Erwerbssystems und der sozialen Sicherung zu konstatieren (Palier/Thelen 2010). Der Ausgangspunkt dieser Entwicklung wird in den industriellen Beziehungen, d.h. in der erfolgreichen Verteidigung der Sicherheiten eines - freilich kleiner werdenden - Kerns von Beschäftigten gesehen, wobei die Abwälzung der Unsicherheit auf den Rand von den Interessenvertretungen der Beschäftigten billigend in Kauf genommen oder gar aktiv betrieben werde; im Ergebnis verlieren damit die für den Kern getroffenen Regelungen ihre Ausstrahlungs- und Leitbildfunktion auf das Erwerbssystem insgesamt, die sie in der Blütezeit des wohlfahrtsstaatlichen Industriekapitalismus einmal hatten. Zweifellos lassen sich vielfältige Phänomene anführen, die in dieses Raster passen, während jedoch diejenigen, die nicht hineinpassen, nicht behandelt werden. Die tautologische Konstruktionsweise von Dualismus-Theorien wird leicht übersehen: Alles, was sich entweder als geschützt oder als prekär klassifizieren lässt, passt auf die eine oder die andere Seite und scheint die „Theorie“ zu bestätigen.

Der Dualisierungsansatz wird der Komplexität der Veränderungen im Erwerbssystem nicht gerecht. Erstens sind die Erwerbssituationen und Lebenslagen der angeblichen Outsider außerordentlich heterogen. Wer in einer Dimension als Outsider erscheint, mag in einer anderen Insider sein; über die kategoriale Feststellung von institutionellen Einschlüssen und Ausschlüssen allein lässt sich kein konsistentes Bild der Erwerbsgesellschaft gewinnen. Zweitens aber wird unterstellt, dass infolge oder mithilfe der „Dualisierung“ der geschützte und privilegierte Kern weitgehend ungeschoren davonkomme. Auch hierbei wird wieder rein institutionell argumentiert, sodass die tief greifenden Veränderungen in den Beschäftigungsbeziehungen gerade auch des angeblichen Kerns ausgeblendet bleiben.

Tatsächlich aber beobachten wir tief greifende Veränderungen auch und gerade beim stabil beschäftigten Kern. Das wi- dersprüchliche Bild einer Stabilisierung von Beschäftigung in rückschauender Betrachtung bei gleichzeitiger Zunahme perspektivischer Unsicherheit lässt sich dahingehend deuten, dass die einstmals für selbstverständlich genommene Beschäftigungssicherheit immer voraussetzungsvoller wird. Zum einen bedeutet das Eindringen marktförmiger Koordinationsmechanismen in die Organisationen, dass größere Teile der Arbeitnehmerschaft ständig und unmittelbar mit den wirtschaftlichen Voraussetzungen des „Überlebens“ ihres Arbeitsplatzes konfrontiert sind. Zum anderen wird die Fortsetzung stabiler Beschäftigungsmuster immer wieder neu gegen Zugeständnisse der Beschäftigten verhandelt, wodurch die Geltung einstmals selbstverständlicher Grundsätze einen immer nur vorläufigen, befristeten Charakter erhält. Die Stabilisierung von Beschäftigung bei subjektiv gesteigerter Unsicherheit garantiert einen umfassenderen, flexibleren und unmittelbareren Zugriff auf Arbeitskraft als im Alternativfalle externnumerischer Flexibilisierung, die den Charakter einer Drohkulisse annimmt, mit der die interne Flexibilisierung durchgesetzt wird. Vor diesem Hintergrund erscheinen die extremen internen Anpassungsleistungen der Unternehmen, die das Durchschlagen der Finanz- und Wirtschaftskrise auf den Arbeitsmarkt verhindert haben, durchaus ambivalent: Ohne die gesteigerte Konzessionsbereitschaft der Beschäftigten wären sie nicht möglich gewesen.

In dem in rückschauender Betrachtung stabilen, perspektivisch aber zunehmend verunsicherten Kern werden immer höhere Preise für diese Stabilität gezahlt. Der umfassendere und zunehmend grenzen- und bedingungslose Zugriff der Betriebe auf ihre Arbeitskräfte überfordert wachsende Teile von ihnen. Die vielfältigen Hinweise auf die Zunahme psychischer Erkrankungen unter Beschäftigten (u. a. Badura et al. 2010), die öffentliche Thematisierung der Burnout-Syndrome Prominenter und der Boom im Bereich professioneller Kompensationsangebote (Wellness, Coaching, Mentaltraining) dürften dabei nicht allein auf steigende Arbeitsbelastungen (Lenhardt et al. 2010) zurückzuführen sein, sondern auch auf ein wachsendes Missverhältnis von Arbeitsanforderungen und Gratifikationen materieller wie immaterieller Art sowie auf die wahrgenommene Ausweglosigkeit beruflicher Situationen (Siegrist/Dragano 2008). Im Verhältnis zur ursprünglichen Ausbildung unterwertige Beschäftigung, die bereits 2006 ein erhebliches Ausmaß angenommen hatte, ${ }^{11}$ führt zu geringerer Zufriedenheit mit Art und Inhalt der Tätigkeit und stellt daher einen Faktor der Störung von BelastungsGratifikations-Bilanzen dar.

\section{7 \\ Schlussfolgerungen für die Arbeitsmarktpolitik}

Arbeitsmarktpolitik ist wesentlich die Förderung von Übergängen am Arbeitsmarkt. Die Eingliederung von Arbeitslosen in Erwerbstätigkeit stellt nur einen solchen Übergang dar. Die Verengung der Arbeitsmarktpolitik auf Arbeitslose und Hilfebedürftige verschenkt Eingliederungsmöglichkeiten, die sich aus Mobilitätsketten ergeben, die ihren Anfang bei bereits Beschäftigten nehmen. Eine aktive Arbeitsmarktpolitik, die durch Auffrischungs-, Anpassungs- und Aufstiegsqualifizierung für Beschäftigte und von Arbeitslosigkeit bedrohte Mobilitätsketten in höherwertige Beschäftigung auslöst, würde gleich zwei Fliegen mit einer Klappe schlagen: Sie würde dem Fachkräftemangel entgegenwirken, und sie würde Beschäftigungsmöglichkeiten für bisher am Arbeitsmarkt Chancenlose eröffnen, die - auch mit passgenauer Förderung - in freiwerdende Positionen aufrücken könnten (Knuth 2011).

Aufwärtsmobilität am Arbeitsmarkt setzt voraus, dass die Abwärtsspiralen bei betrieblichen Einstiegspositionen gestoppt werden. Anscheinend sind die Betriebe dazu - außer bei wenigen hochspezialisierten Funktionen - nicht in der Lage. Ein gesetzlicher Mindestlohn könnte stabilisierend auf das Lohngefüge insgesamt wirken, was vermutlich auch der Grund dafür ist, dass er so vehement bekämpft wird. Eine Mobilität, die die Allokation von Arbeitskraft verbessert und die Motivation der Arbeitskräfte steigert, liegt offensichtlich im gesamtwirtschaftlichen Interesse, aber zunächst nicht im einzelwirtschaftlichen. Die Arbeitsmarktpolitik muss wieder dazu kommen, gesamtwirtschaftliche Rationalitäten durchzusetzen statt einzelwirtschaftlichen Wünschen nachzulaufen.

11 Betroffen waren 15,2 \% der Frauen und 9,5 \% der Männer zwischen 18 und 40 Jahren (Hall 2010). 
Die „aktivierende“ Arbeitsmarktpolitik hat dazu beigetragen, Übergänge aus Arbeitslosigkeit in Beschäftigung zu beschleunigen. Wahrscheinlich hat dieses - trotz der in Rechnung zu stellenden Drehtüreffekte - per saldo auch zum Abbau von Arbeitslosigkeit beigetragen. Die deutsche Arbeitsmarktpolitik hat dieses Ergebnis allerdings mehr durch „Fordern“ als durch „Fördern“ erreicht. „Fordern“ wirkt bei denjenigen, die die Ressourcen haben, den Forderungen nachzukommen; diejenigen mit ernsthaften Vermittlungshemmnissen bleiben zurück. Die mit den Hartz-Reformen verbundene Botschaft, dass Arbeitslosigkeit Faulheit sei, auf die es kein Recht gebe (Gerhard Schröder), und der dementsprechende Rückbau der Arbeitslosenversicherung (bzw. die Abschaffung der versicherungsähnlich ausgestalteten Anschlussleistung) scheinen sich auf den Arbeitsmarkt insgesamt lähmend ausgewirkt zu haben. Beschäftigte scheuen das Risiko eines Arbeitgeberwechsels, weil sie wissen, dass die relative Absicherung ihrer Einkommensposition nur kurz dauert, falls auf dem Weg über den Arbeitsmarkt etwas schief geht. Mit dem Wegfall der Entgeltsicherung für Ältere ab 2012 (Dietz et al. 2011) wird der Weg der Rücknahme von mobilitätsförderlichen Sicherheitsgarantien zumindest symbolisch - die faktische Inanspruchnahme des Instruments war gering - weiter verfolgt. Statt des immer weiteren Rückbaus der Arbeitslosenversicherung auf Kosten des Steuerzahlers wäre ein moderater Ausbau zu empfehlen, durchaus verbunden mit Anreizelementen, aber mit der Perspektive einer längeren, von Bedürftigkeit unabhängigen maximalen Leistungsdauer (Brussig/Knuth 2011). Dadurch würde die derzeit eklatante Unausgewogenheit von Versicherung und Grundsicherung korrigiert und verhindert, dass das Regime der Grundsicherung letztlich die gesamte Arbeitsmarktpolitik und die Wahrnehmung der Beschäftigten vom Arbeitsmarkt dominiert.

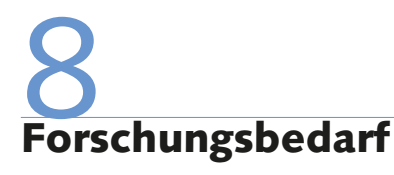

Die vorstehenden Überlegungen stellen den Versuch dar, disparate und auf den ersten Blick widersprüchliche Befunde in einen Zusammenhang zu bringen, der beim derzeitigen Stand des Wissens nur hypothetischen Charakter haben kann. Daraus ergeben sich Forschungsfragen, von denen hier nur einige angerissen werden können.

Zunächst wäre zu fragen, ob die deskriptiv getroffene Feststellung, dass der Arbeitsmarkt insgesamt zähflüssiger geworden sei, bei Kontrolle für strukturelle Veränderungen zutrifft. Demografische Alterung (Schneider 2007) und Zunahme von Zwei-Verdiener-Haushalten auch mit Kindern sind sicherlich mobilitätsdämpfende Entwicklungen, aber diese sollten zumindest teilweise kompensiert werden durch Entwicklungen auf der Nachfrageseite wie Tertiarisierung, Abnahme durchschnittlicher Betriebsgrößen und Zunahme atypischer Beschäftigungsformen. Doch selbst wenn sich abnehmende Fluktuation vollständig durch strukturelle Veränderungen auf der Angebots- und der Nachfrageseite erklären ließe, wäre das kein Grund zur Beruhigung, sondern Anlass über Maßnahmen zur Förderung beruflicher Mobilität nachzudenken.

Sollte sich jedoch die hier getroffene Annahme bestätigen, dass institutionelle Veränderungen des Arbeitsmarktregimes zu einem veränderten Verhalten der Arbeitsmarktakteure geführt haben, so wäre weiter zu fragen, ob die leichte Zunahme von Personalrekrutierungen aus dem Kreis der Arbeitslosen über die Verkürzung der Vakanzketten die Abnahme der Fluktuation erklären kann (und dann gewissermaßen der hinzunehmende Preis für die Integration von mehr Arbeitslosen in Beschäftigung wäre), oder ob die Arbeitsmarktreformen darüber hinaus die
Risikoaversion der Beschäftigten erhöht haben. Alternativ wäre zu untersuchen, ob der Eindruck zutrifft, dass die Einstiegskonditionen bei Neueinstellungen oder die Aufstiegschancen aus solchen Einstiegspositionen schlechter geworden sind, und es wäre zu fragen, warum die Betriebe bei der Gestaltung dieser Einstiegskonditionen nicht auf die Veränderung der Angebots-Nachfrage-Relationen reagieren bzw. lange Zeit nicht reagiert haben. In diesem Zusammenhang wäre schließlich zu untersuchen, ob Personen, die aus Arbeitslosigkeit Beschäftigung aufgenommen haben, hierbei gegenüber ihrer früheren Beschäftigung größere Lohnzugeständnisse gemacht haben als es vor den Hartz-Reformen der Fall war. Insofern wären vorliegende Selbstauskünfte zur Konzessionsbereitschaft (Bender et al. 2008) zu überprüfen durch die Analyse der Konditionen, zu denen tatsächlich Beschäftigung aufgenommen wurde.

Schließlich wäre die wachsende Rolle der Arbeitnehmerüberlassung als intermediärer Dienstleistung am Arbeitsmarkt zu untersuchen: Welchen Anteil hat sie an der Steigerung von Einstellungschancen Arbeitsloser einerseits, der möglichen Verschlechterung von Einstiegskonditionen andererseits? Verfälscht sie möglicherweise die Wahrnehmung der Betriebe vom Arbeitsmarkt und verzögert deren Anpassungsreaktionen an veränderte Knappheitsverhältnisse? Wie wirkt sich die zunehmende Nutzung der Arbeitnehmerüberlassung als Vermittlungskanal der Arbeitsverwaltung auf die Wahrnehmung von Chancen und Risiken des Arbeitsmarktes durch veränderungsbedürftige Beschäftigte und durch Arbeitslose aus?

Letztlich geht es darum, die Wirkungen von Arbeitsmarktreformen nicht nur bezogen auf einzelne Instrumente und die jeweiligen Zielpersonen, sondern im Hinblick auf die Funktionsweise des Arbeitsmarktes insgesamt zu bestimmen und zu bewerten - eine Betrachtungsweise, die derzeit weitgehend fehlt. 


\section{LITERATUR}

Achatz, J./Trappmann, M. (2009): Wege aus der Grundsicherung. Befragung von Arbeitslosengeld-II-Beziehern, IAB-Kurzbericht (28), Nürnberg

Badura, B./Ducki, A./Schröder, H./Klose, J./Macco, K. (2010):

Fehlzeiten-Report 2009. Arbeit und Psyche: Belastungen reduzieren Wohlbefinden fördern, Berlin/Heidelberg

Bender, S./Koch, S./Mosthaf, A./Walwei, U. (2008): Paradigmenwechsel in der Arbeitsmarktpolitik. Wirkt die Aktivierung auf die Konzessionsbereitschaft?, in: Sozialer Fortschritt 57 (3), S. 75-84

Boerner, S./Schramm, F. (1998): Fluktuationsneigung in den neunziger Jahren: Eine empirische Untersuchung anhand des Sozio-ökonomischen Panels, in: Zeitschrift für Personalforschung 12 (1), S. 79-97

Bohulsky, Y./Erlinghagen, M./Scheller, F. (2011): Arbeitszufriedenheit in Deutschland sinkt langfristig. Auch geringe Arbeitszufriedenheit im europäischen Vergleich, IAQ-Report 2011-03, Duisburg

Brussig, M./Knuth, M. (2011): Reformoptionen in der "Grundsicherung für Arbeitsuchende“. Individualisieren, konzentrieren, intensivieren, Expertise im Auftrag der Abteilung Wirtschafts- und Sozialpolitik der Friedrich-Ebert-Stiftung Bonn, WISO Diskurs, Bonn

Bundesagentur für Arbeit (2011a): Arbeitsmarkt 2010, Bundesagentur für Arbeit, Nürnberg, Amtliche Nachrichten der Bundesagentur für Arbeit 58 (Sondernummer 2), Nürnberg

Bundesagentur für Arbeit (2011b): Sockel- und Langzeitarbeitslosigkeit, Bundesagentur für Arbeit Nürnberg, Arbeitsmarktberichterstattung, Nürnberg

Dietz, M./Koch, S./Krug, G./Stephan, G. (2011): Die Entgeltsicherung für Ältere: ein Auslaufmodell?, in: WSI-Mitteilungen, 64 (5),

S. $226-233$

Dlugosz, S./Stephan, G./Wilke, R. (2009): Verkürzte Bezugsdauern für Arbeitslosengeld: Deutliche Effekte auf die Eintritte in Arbeitslosigkeit, IAB-Kurzbericht (30), Nürnberg

Doeringer, P./Piore, M. (1971): Internal labor markets and manpower adjustment, New York

Eichhorst, W./Marx, P./Thode, E. (2009): Arbeitsmarkt und Beschäftigung in Deutschland 2000-2009. Benchmarking Deutschland: Beschäftigungserfolge bei zunehmender Differenzierung, Bertelsmann Stiftung, Gütersloh

Erlinghagen, M. (2010): Mehr Angst vor Arbeitsplatzverlust seit Hartz? Langfristige Entwicklung der Beschäftigungsunsicherheit in Deutschland, IAQ-Report 2010-02, Duisburg

Erlinghagen, M./Wagner, G. (2010): Die Angst vor Jobverlust verursacht hohe Kosten, in: Handelsblatt vom 6.5., S. 7

European Commission, Directorate-General for Employment, Social Affairs \& Equal Opportunities (2010): Employment in Europe 2010, Brüssel

Fachinger, U./Himmelreicher, R. (2007): Alterslohnprofile und Qualifikation in den alten Bundesländern, in: Deutsche Rentenversicherung 62 (11/12), S. 750-770

Fehr, S./Vobruba, G. (2011): Die Arbeitslosigkeitsfalle vor und nach der Hartz-IV-Reform, in: WSI-Mitteilungen 64 (5), S. 211-217

Fietze, S. (2011): Arbeitszufriedenheit und Persönlichkeit: „Wer schaffen will, muss fröhlich sein!“, SOEPpapers on multidisciplinary panel data research (388), Berlin

Graf, T./Rudolph, H. (2009): Viele Bedarfsgemeinschaften bleiben lange bedürftig. Dynamik im SGB II 2005-2007, Institut für Arbeitsmarktund Berufsforschung, IAB-Kurzbericht (5), Nürnberg
Hall, A. (2010): Arbeiten Frauen und Männer unter ihrem Wert? Zum Einfluss dualer und schulischer Ausbildungsberufe auf ausbildungsinadäquate Erwerbstätigkeit in Deutschland, in: Schweizerische Zeitschrift für Soziologie 36 (1), S. 131-159

Hauser, F./Schubert, A./Aicher, M. (2008): Unternehmenskultur, Arbeitsqualität und Mitarbeiterengagement in den Unternehmen in Deutschland. Ein Forschungsprojekt des Bundesministeriums für Arbeit und Soziales, Durchführung: psychonomics AG in Kooperation mit dem Institut für Wirtschafts- und Sozialpsychologie der Universität zu Köln und freundlicher Genehmigung des Great Place to Work® Institute Herzog-Stein, A./Seifert, H. (2010): Der Arbeitsmarkt in der Großen Rezession - Bewährte Strategien in neuen Formen, in: WSI-Mitteilungen 63 (11), S. 551-559

Hohendanner, C. (2010): Unsichere Zeiten, unsichere Verträge? Befristete Arbeitsverträge zwischen Auf- und Abschwung, IAB-Kurzbericht (14), Nürnberg

Horn, G./Logeay, C./Zwiener, R. (2008): Wer profitierte vom Aufschwung?, IMK-Report (27), Düsseldorf

Kalina, T./Weinkopf, C. (2010): Niedriglohnbeschäftigung 2008: Stagnation auf hohem Niveau - Lohnspektrum franst nach unten aus, IAQ-Report (6), Duisburg

Keller, B./Seifert, H. (2011): Atypische Beschäftigungsverhältnisse. Stand und Lücken der aktuellen Diskussion, in: WSI-Mitteilungen 64 (3), S. 138-145

Kerr, C. (1954): The balkanization of labor markets, in: Bakke, E. W./ Hauser, P. M./Palmer, G. L./Myers, C. A.; Yoder, D./Kerr, C. (Hrsg.): Labor mobility and economic opportunity. Cambridge/New York, S. 92-110 Knuth, M. (2006): „Hartz IV“ - die unbegriffene Reform. Wandel der Erwerbsordnung durch Verallgemeinerung des Fürsorge-Regimes, in: Sozialer Fortschritt 55 (7), S. 160-168

Knuth, M. (2010): Fünf Jahre Hartz IV: Zwischenbilanz und Reformbedarf, in: Orientierungen zur Wirtschafts- und Gesellschaftspolitik 123, S. $14-23$

Knuth, M. (2011): Schriftliche Stellungnahme zur öffentlichen Anhörung von Sachverständigen in Berlin am 5. September zum Entwurf eines Gesetzes zur Verbesserung der Eingliederungschancen am Arbeitsmarkt, Bundestags-Ausschuss-Drucksache 17(11)610, Berlin Koller, L./Rudolph, H. (2011): Viele Jobs von kurzer Dauer. Arbeitsaufnahmen von SGB-II-Leistungsempfängern, IAB-Kurzbericht (14), Nürnberg

Koslowski, A./Konle-Seidl, R./Eichhorst, W./Marx, P. (2011): The changing nature of transitions between non-employment and employment in West European labour markets, Paper presented at the 9th Annual Espanet Conference, 8-10 September, Stream 6: Labour market policy, activation and beyond, Valencia

Lenhardt, U./Ertel, M./Morschhäuser, M. (2010): Psychische Arbeitsbelastungen in Deutschland: Schwerpunkte - Trends - betriebliche Umgangsweisen, in: WSI-Mitteilungen 63 (7), S. 335-342

Lindbeck, A./Snower, D. J. (1988): The insider-outsider theory of employment and unemployment, Cambridge

Matiaske, W./Mellewigt, T. (2001): Arbeitszufriedenheit: Quo vadis? Eine Längsschnitt-Untersuchung zu Determinanten und zur Dynamik von Arbeitszufriedenheit, in: Die Betriebswirtschaft 61 (1), S. 7-24 Möller, J. (2010): The German labor market response in the world recession - de-mystifying a miracle, in: Zeitschrift für ArbeitsmarktForschung 42 (4), S. 325-336 
Orlowski, R./Riphahn, R. (2008): Seniority in Germany: New evidence on returns to tenure for male full-time workers, in: Zeitschrift für ArbeitsmarktForschung 41 (2/3), S. 139-155

Orlowski, R./Riphahn, R. (2011): Lohnentwicklung im Lebenszyklus. Eine Analyse von Ausmaß, Begründung und Heterogenität von Lohnsteigerungen, in: Zeitschrift für ArbeitsmarktForschung 44 (1/2), S. $29-41$

Palier, B./Thelen, K. (2010): Institutionalizing dualism: Complementarities and change in France and Germany, in: Politics and Society 38 (1), S. $119-148$

Rebien, M./Kettner, A. (2011): Die Konzessionsbereitschaft von Bewerbern und Beschäftigten nach den Hartz-Reformen, in: WSI-Mitteilungen 64 (5), S. 218-225

Rhein, T. (2010): Ist Europa auf dem Weg zum "Turbo-Arbeitsmarkt"? Beschäftigungsdynamik im internationalen Vergleich, IAB-Kurzbericht (19), Nürnberg

Schettkat, R. (1996): Flows in labour markets. Concepts and international comparative results, in: Ders. (Hrsg.): The flow analysis of labour markets, Routledge studies in the modern world economy No. 3, London
Schmid, G. (2008): Von der Arbeitslosen- zur Beschäftigungsversicherung. Wege zu einer neuen Balance individueller Verantwortung und Solidarität durch eine lebenslauforientierte Arbeitsmarktpolitik, Gutachten für die Friedrich-Ebert-Stiftung, WISO Diskurs, Berlin Schmid, G. (2011): Übergänge am Arbeitsmarkt. Arbeit, nicht nur Arbeitslosigkeit versichern, Berlin

Schneider, L. (2007): Zu alt für einen Wechsel? Zum Zusammenhang von Alter, Lohndifferentialen und betrieblicher Mobilität, in: Sozialer Fortschritt 56 (7-8), S. 180-186

Siegrist, J./Dragano, N. (2008): Psychosoziale Belastungen und Erkrankungsrisiken im Erwerbsleben. Befunde aus internationalen Studien zum Anforderungs-Kontroll-Modell und zum Modell beruflicher Gratifikationskrisen, in: Bundesgesetzblatt - Gesundheitsforschung - Gesundheitsschutz 51, S. 305-312

Trischler, F./Kistler, E. (2010): Arbeitsbedingungen und Erwerbsverlauf. Arbeitspapier 2 des Forschungsprojektes "Gute Erwerbsbiographien“, Düsseldorf/Stadtbergen 